\title{
Cellular MYCro Economics: Balancing MYC Function with MYC Expression
}

\author{
David Levens \\ Laboratory of Pathology, Center for Cancer Research, National Cancer Institute, Bethesda, \\ Maryland 20892-1500 \\ Correspondence: levens@helix.nih.gov
}

The expression levels of the MYC oncoprotein have long been recognized to be associated with the outputs of major cellular processes including proliferation, cell growth, apoptosis, differentiation, and metabolism. Therefore, to understand how MYC operates, it is important to define quantitatively the relationship between MYC input and expression output for its targets as well as the higher-order relationships between the expression levels of subnetwork components and the flow of information and materials through those networks. Two different views of MYC are considered, first as a molecular microeconomic manager orchestrating specific positive and negative responses at individual promoters in collaboration with other transcription and chromatin components, and second, as a macroeconomic czar imposing an overarching rule onto all active genes. In either case, $c-m y c$ promoter output requires multiple inputs and exploits diverse mechanisms to tune expression to the appropriate levels relative to the thresholds of expression that separate health and disease.

$M$ YC was first discovered as the transforming gene of avian retrovirus MC29 (Wasylishen and Penn 2010; Eisenman 2013). It was subsequently shown to be a nuclear DNA-binding protein of cellular origin (Abrams et al. 1982; Moelling et al. 1982; Vennstrom et al. 1982). Further links between MYC and cancer were forged as $c-m y c$ was shown to be translocated with immunoglobulin genes in Burkitt lymphoma (human) and plasmacytoma (mouse) (Boxer and Dang 2001; Janz 2006), chromosomally amplified or activated by viral insertions such as occurs in cervical carcinoma with human papillomavirus (HPV) (see, e.g., Alitalo et al. 1983; Peter et al. 2006). Chromosomal aberrations of MYC most often leave protein coding sequences intact. Unlike other proto-oncogenes, MYC requires no changes in protein sequence to become oncogenic; the proto-oncogene becomes the oncogene through abnormal expression (Love et al. 2012). Although coding mutations do occur, they mostly influence the stability and turnover of the protein, thereby increasing its levels rather than modifying fundamental MYC action. MYC oncogenesis would seem to be more a matter of degree than of the quality of MYC function (Liu and Levens 2006; Wierstra and Alves 2008; Levens 2010).

Lots of direct and circumstantial evidence indicates that MYC levels matter. Small changes in MYC levels drive cells from cell-cycle arrest to proliferation or across thresholds that trigger

Editors: Chi V. Dang and Robert N. Eisenman

Additional Perspectives on MYC and the Pathway to Cancer available at www.perspectivesinmedicine.org

Copyright (C) 2013 Cold Spring Harbor Laboratory Press; all rights reserved; doi: 10.1101/cshperspect.a014233

Cite this article as Cold Spring Harb Perspect Med 2013;3:a014233 


\section{Levens}

apoptosis (Murphy et al. 2008; Shachaf et al. 2008). Whereas normal diploid human fibroblasts are immortalized by retrovirally transduced telomerase, after the knockout of one $c$-myc allele, these cells instead senesce (Guney et al. 2006). Cell size and indeed whole organism size are sensitive to twofold changes in MYC (Iritani and Eisenman 1999; Johnston et al. 1999; Schuhmacher et al. 1999; Trumpp et al. 2001). Using elegant genetic tricks, developmental compartments in Drosophila were populated with admixtures of normal cells and cells expressing either twice or half of the normal amounts of MYC. In each case, the low-MYC cells were killed as the high-MYC cells expanded, refilled the compartment, and developed normally (de la Cova et al. 2004; Moreno and Basler 2004; Johnston 2013). If MYC levels are so important, what does it do mechanistically that makes cells so sensitive to its measure, and how are MYC levels controlled?

\section{DNA BINDING BY MYC}

MYC encodes a bHLH-ZIP (basic helix-loophelix-leucine zipper protein) transcription factor (TF) that dimerizes with another member of the same family, MAX. This heterodimer constitutes a DNA-binding domain with maximal selectivity for the sequence CACGTG (E-box) (Blackwood and Eisenman 1991), although interactions with noncanonical E-box sequences also occur. The measured dissociation constant for recombinant MYC-MAX with E-boxes in naked DNA is $\sim 100 \mathrm{~nm}$, not especially strong if this number reflects the intracellular $K_{d}$, and it is weaker than some other bHLH-ZIP dimers (Park et al. 2004). The ratio of specific to nonspecific DNA binding by MYC-MAX is a missing biophysical parameter that is required to model MYC specificity and function.

In crystals, two MYC-MAX heterodimers dimerize, in turn forming a heterotetramer with two diametrically opposed bound E-boxes. Simultaneous engagement of both DNA-binding domains in vivo to the same segment of DNA would create a topologically closed MYC-MAX loop, an arrangement replete with regulatory implications and inviting speculation about mechanisms to control gene activity (Nair and Burley 2003). However, later experiments failed to detect or directly visualize such loops (Lebel et al. 2007). Moreover, mice heterozygous for fully functional carboxy-terminally tagged MYC-EGFP knock-in and wild-type MYCalleles show no evidence of tetramer formation. If expressed at equal levels, $50 \%$ of such tetramers should include one molecule each of MYCEGFP and MYC, so immunoprecipitation using anti-EGFP would be expected to coimmunoprecipitate MYC; but with the separate formation of MYC-EGFP/MAX or MYC/MAX heterodimers, such coimmunoprecipitation would not occur. Indeed, extracts from serum-stimulated heterozygous fibroblasts showed no MYCEGFP/MYC coimmunoprecipitation, indicating that tetramerization must require special circumstances if it were to occur in vivo (Nie et al. 2012).

MYC interacts with a large number of proteins and multicomponent complexes. Particular attention has been focused on MYC partners with obvious connections to gene expression, especially those directly participating in the regulation of transcription and chromatin (Amati et al. 2001; Cowling and Cole 2006; Agrawal et al. 2010; Kim et al. 2010). MYC's accomplices include complexes bearing the histone acetylases GCN5 or NuA4, the chromatin remodeler SWI/SNF, general transcription factors (TFs) TFIIH, TBP, and sequence-specific TFs such as YY1 (Shrivastava et al. 1996; Cowling and Cole 2007). However, a functionally unbiased roster of MYC partners includes representatives of diverse sets of regulatory, structural, and catalytic proteins that are involved in a variety of subcellular processes and networks (Agrawal et al. 2010). It is not known whether MYC projects its protean influence as a component of regulatory complexes by modulating the activities of other complex subunits or whether these interactions act as a sink competing against a smaller number of true MYC effectors thereby modulating the levels of active MYC.

\section{WHAT DOES MYC DO?}

Extensive literature associates changes in MYC levels, either through pathologic activation or 
experimental manipulation, with altered outputs of many pathways and regulatory networks, including proliferation; cell-cycle, protein, and RNA biosynthesis; metabolism; and signaling (Eilers and Eisenman 2008; Dang 2010, 2012; Wasylishen and Penn 2010). Careful studies have failed to define a consistent and coherent set of MYC targets that channel MYC's output onto these processes. Most studies attempting to define a pathway that conducts MYC's oncogenic impulse through an explicit series of direct and indirect downstream effectors have been disappointing. However, one recent report indicates that MYC-mediated down-regulation of tristetraprolin (TTP), a protein that destabilizes mRNAs through $3^{\prime}$-UTR (untranslated region) AU-rich elements (AREs), up-regulates hundreds of targets. That TTP is necessary, but not sufficient, for tumorigenesis reveals that the genomic realm of MYC exceeds that of TTP (Ji et al. 2011). So, two views of MYC action have emerged. First, although MYC regulates a large set of targets, camouflaged within this set is a cadre of molecular conspirators that hijack the cell and cause cancer. Second, MYC by itself drives the general activation of all cellular processes reinforcing or exaggerating only those responses already programmed by the cell. In the first scenario, MYC acts digitally, throwing key switches; in the second, it is an analog amplifier and any changes in cellular state arise secondarily from preprogrammed input-output transfer functions.

MYC targets may be operationally defined by genomic DNA-binding differences or expression changes associated with changes in MYC levels. Each of these definitions suffers practical and conceptual limitations. DNA binding, in vivo, need not be functionally relevant; all TFs must discriminate between bona fide cognate sites that have evolved to channel TF activity and the vastly greater number of nonspecific sites that have been presumed to be biologically inert. Thus, MYC association with a region of active chromatin might indicate that MYC participates in the genetic processes programmed by these regions, or it might simply reflect the nonspecific association of MYC with the most accessible segments of the genome. If it is the latter, then many, if not most, MYC-binding sites might be physiologically and pathologically irrelevant. If MYC binds with high selectivity at true target sequences, those sites should saturate at much lower levels of MYC than required to populate the low-affinity or genomewide nonspecific binding sites to any degree. In fact, the binding distribution of MYC at all of its sites across the genome is distinctly unimodal with no high-affinity tail or peak. Therefore, any threshold used to discriminate specific versus nonspecific binding by MYC is arbitrary (Nie et al. 2012). Binding patterns alone seemed not to spotlight MYC action if gauged by relative, rather than absolute, changes in expression.

The genomic targets of MYC may also be defined by expression, that is, the identification of genes whose expression is increased or decreased in the presence of MYC. Defining MYC targets by expression suffers from issues intrinsic to the measurement of mRNA levels and the comparison of samples. These issues include the choice of thresholds to justify the moniker "MYC-regulated." Most often, equal amounts of RNA (total or poly[A]) are compared among samples, and the relative changes are noted. In this situation, a threshold demarcates statistical significance and only those targets surmounting the threshold are evaluated further; the more stringent the threshold, the fewer targets recovered. As always, this trade-off between specificity and sensitivity is a problem. This analysis implicitly assumes that the compared cells contain equal amounts of RNA. Sometimes these analyses highlight important biological and mechanistic differences, but in other situations this normalization may obscure global up- or down-regulation of cellular contents among cells with more or less contents. For example, if MYC were a universal activator, normalization to the mean or median of cellular total RNA or mRNA would make genes that are actually upregulated, but less than the mean/median appear to be down-regulated. In fact, adjusting for total RNA greatly shrinks the set of MYCrepressed mRNAs - so much so that it becomes a contentious issue as to whether any targets are directly repressed by MYC (Lin et al. 2012; Loven 


\section{Levens}

et al. 2012; Nie et al. 2012). One view holds that MYC is fundamentally an activator, and therefore any apparent direct repression is either a normalization-accounting issue or derives from other gene regulatory mechanisms evolved to override MYC's action. A contrasting view is that MYC activity is context dependent and that MYC itself interacts with transcription and/or chromatin components to direct decreased expression depending on the sequence and chromosomal environment of a particular target (Eilers and Eisenman 2008).

Whether defined by binding or by expression, experiments identifying MYC targets have interrogated a wide range of cell and experimental systems, most often using cancer cell lines expressing pathologically deregulated MYC or experimental systems that express MYC nonphysiologically. Fewer studies have attempted to enumerate the physiological targets of MYC. The diversity of these systems has made it difficult to deconvolute the experimental and biological heterogeneity of this data. To hone in on true MYC biology, informatic approaches have been used to enumerate a "MYC signature"-genes that are unequivocally induced or repressed by MYC and should prove to be reliable markers of MYC expression while providing insight into the biological processes under MYC's purview (Shaffer et al. 2006; Margolin et al. 2009; Wasylishen and Penn 2010; Ji et al. 2011). The signature genes recovered from these attempts incompletely populated diverse pathways, molecular structures, and cellular processes that appeared to be related to the increase in biomass demanded as cells enlarge ( $\mathrm{Ji}$ et al. 2011).

To distill MYC function, it is important to attempt to separate the direct and indirect effects of its activity. Direct targets serve as MYC's agents to alter the expression of its indirect targets. Determining whether MYC changes a gene's mRNA level by binding to that gene's regulatory sequences and modifying transcription, or indirectly by adjusting the levels or activities of other TFs that bind to the gene's regulatory sequences is a difficult problem (Yap et al. 2011). Moreover, in the case of feed-forward regulation, a target may be simultaneously direct and indirect (Alon 2007). One way to discriminate direct versus indirect is to use posttranscriptionally activatable MYC-ER $\mathrm{t}_{\mathrm{t}}$ a fusion of MYC with the ligand-binding domain of a tamoxifenspecific estrogen receptor (Eilers et al. 1989; Littlewood et al. 1995). Adding tamoxifen and monitoring ligand-induced gene expression in the presence or absence of protein synthesis inhibitors such as cycloheximide may separate direct and indirect targets. Unfortunately because of its short half-life, MYC action cannot be sustained in the absence of new protein synthesis. Furthermore, many mRNAs, especially those bearing AREs, are stabilized by cycloheximide, making it difficult to discriminate changes resulting from MYC versus translation inhibition. Nevertheless, applying rigorous criteria for expression changes in HO15.19 Myc-ER $_{t}$ cells activated in the presence or absence of cycloheximide identified 21 definitive direct targets, and all 21 were up-regulated (O'Connell et al. 2003). These results are consistent with the notion that MYC is fundamentally an activator and that MYC repression in good measure works through its indirect targets. The identification of the repressive histone methylase Ezh2 as both a direct and indirect MYC target highlights a major pathway for MYC-influenced, but not direct, MYC-driven repression (Nie et al. 2012; Kaur and Cole 2013).

A recent study examining the genomic binding sites of MYC and global gene expression in resting and activated lymphocytes and in embryonic stem cells reevaluated the systems biology of MYC, yielding a model likely to prove at least of heuristic value (Nie et al. 2012). Resting or activated $\mathrm{B}$ cells and $\mathrm{T}$ cells from mice homozygous for fully functional MYC-EGFP knockin alleles were compared for MYC binding, RNA polymerase distribution, and mRNA expression across the genome. Using median-mean normalization and fold-change thresholds to analyze mRNA expression, and imposing stringent thresholds for ChIP-Seq peak detection, returned an ontologically and biochemically complex set of targets as typical for MYC genomic binding and expression experiments. Although the expression of most MYC-binding targets seemed unchanged, of those that 
changed, two-thirds were up-regulated and onethird were down-regulated.

Reanalysis of the same data normalizing to cell number and removing arbitrary thresholds for expression level or for ChIP-seq peak detection yielded a different perspective. Broad coherence between MYC binding and output was evident with this analysis. Across all samples, the logarithm of mRNA expression level correlated with the magnitude of promoter-bound MYC for all genes, implying a MYC-driven universal increase in RNA synthesis. Consistent with such a role, mRNA levels increased upon activating naïve $B$ cells $\sim 2.5$-fold at 12 h postactivation, approaching 15 -fold at full activation; this increase was blocked when MYC was inactivated either by conditional knockout or through inhibition of MYC-MAX dimerization. Without MYC, activation proceeds through only the earliest phases until the point when MYC would be induced. Blocking MYC prevented the expression increase across a panel of randomly selected genes not previously recognized as MYC targets when normalized to the median or mean. Genes already switched off and residing in heterochromatin were refractory to MYC amplification. Inducing an exogenous MYC in a lymphoblastoid cell line also supported the concept that it is a universal activator of transcription (Lin et al. 2012; Loven et al. 2012).

Generally after assembling into a preinitiation complex, RNA polymerase initiates transcription, advances a short distance, and pauses. Reinitiation is thwarted until the paused polymerase is released. The amount of MYC at the promoters of active genes, in activated B cells, reflected the amount of RNA polymerase loaded at each transcription start site. The level of RNA polymerase preloaded in the resting state anticipated the magnitude of MYC recruitment during activation. If promoters recruit MYC according to the intensity of transcriptional output and if MYC increases that output, a positivefeedback loop is created, in principle, describing the relationship between MYC binding and expression and operationally making MYC an exponential amplifier. This positive feedback may be passive in part, owing to increased promoter availability following MYC-enhanced pause re- lease but may indicate that MYC actively facilitates promoter reloading and progression to the pause point (Rahl et al. 2010; Lin et al. 2012; Nie et al. 2012; Rahl and Young 2013).

Thus, we have two fundamentally different views of MYC action (Fig. 1) (Walz and Eilers 2013). In the conventional view, MYC is recruited in a context-dependent manner to particular genes according to the disposition of E-boxes and the spectrum of available TFs and chromatin regulators. In this view, gene-by-gene MYC is helping to set the microeconomic program of the cell, negotiating with its molecular partners at each target whether a gene is on or off and determining the direction (activation vs. repression) and magnitude of promoter output. For each MYC-regulated process, the rate-controlling genes must have evolved the appropriate arrangement of E-boxes and other features to mold the MYC response. In this scheme, the net effect of MYC expression, whether physiological or pathological, is the superimposition of the responses at each promoter; each individual promoter evolves its own MYC transaction more or less apart from the other components of its functional network.

The alternative view is that MYC acts to increase the transcription of genes that are already on, amplifying their output exponentially. In this view, MYC imposes an overarching "macroeconomic" rule on the cell, leaving each network and pathway to reach its own set point according to the new levels of expression of its genes and available resources. Exponential amplification, in which highly expressed genes are relatively more up-regulated than genes with lower expression levels, may be indicative of a positive-feedback process in which MYC increases promoter output. This in turn recruits more MYC, etc., until something becomes limiting. Although MYC is an amplifier and not a specifier of cell state, it can contribute indirectly to reprogramming gene expression by driving repressors, TFs, and signaling systems with nonlinear dose response curves across key thresholds. A rigorous evaluation of the amplifier model demands determination of the feedback and feed-forward relationships among the amplified active genes. 
D. Levens

A

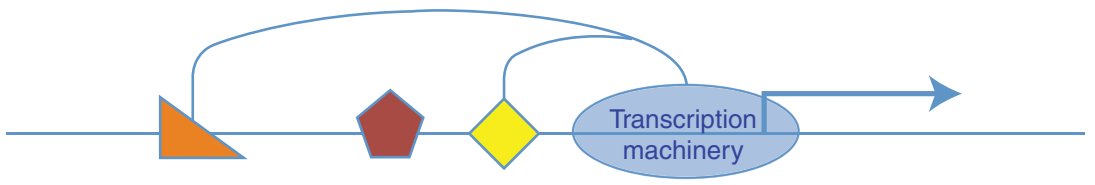

B

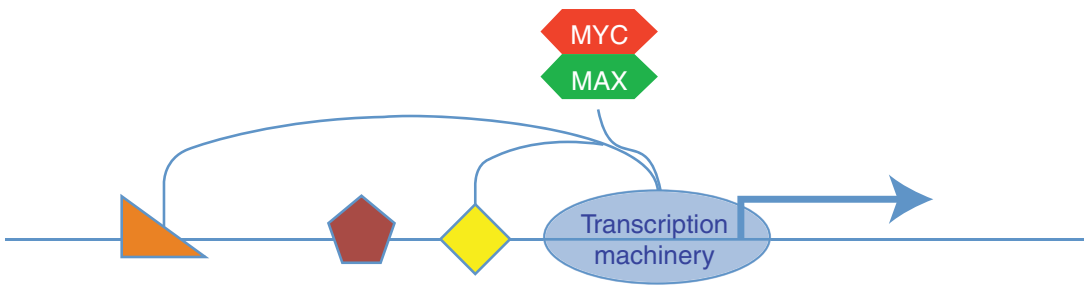

C

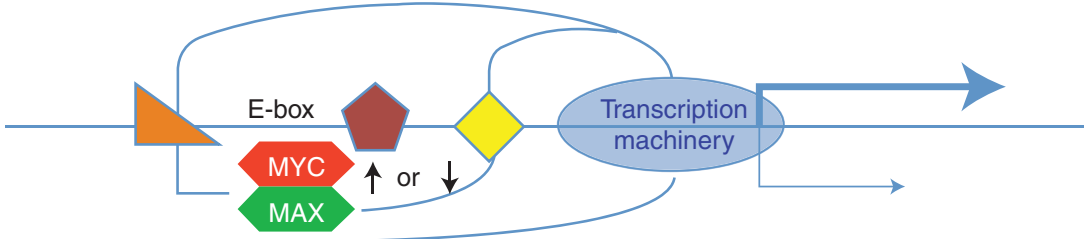

Figure 1. Macroeconomic versus microeconomic models of MYC action. (A) In the absence of MYC, target genes are regulated by the integrated inputs of whatever factors are relevant. The logic of the integration is determined locally to set the output. (B) In the macroeconomic model, MYC is recruited both through DNA and protein interactions with the transcription machinery and acts to augment output according to the preexisting level of expression. MYC augments the outputs of more highly expressed genes more than it does of genes expressed at lower levels. MYC does not directly alter the regulatory logic of the promoter. $(C)$ In the microeconomic model, MYC is recruited via E-boxes and then engages in negotiations with the panel of transcription and chromatin factors bound locally. The output of each promoter is situational and determined locally as MYC may activate or repress to different degrees.

\section{WHY CELLS NEED A NONLINEAR AMPLIFIER}

How can the preferential amplification of highly expressed genes be explained or at least rationalized? Comparison of resting and activated B cells provokes an attractive speculation. A fully activated $B$ cell contains $\sim 550,000$ total mRNAs, whereas a resting cell contains $\sim 35,000$ transcripts. Under both conditions, the mRNAs are derived from $\sim 12,000$ active genes (Kouzine et al. 2013). Thus, the number of transcripts / gene averages $\sim 3$ in a resting cell and $\sim 45$ at full activation. Compared with activated cells, for many if not most genes, expression can be scaled down to the low levels of the naïve lymphocyte. But for those genes already expressed at low levels ( $<10$ mRNAs/cell) in activated cells, a proportionate scaling down to resting levels would leave many genes, presumably some essential for cellular viability, present at less than one transcript cell. To ensure its survival, relative to activated cells, a naïve $B$ cell would need to express relatively more of scarce versus abundant transcripts. Thus, the naïve cell may be thought of as having a logarithmically compressed transcriptome compared with the activated state. An exponential amplifier would expand the transcriptome to functional levels. This hypothesis rationalizes the pulse of MYC expression that occurs almost universally in response to cell activation in metazoan systems as a means to jump-start a quiescent cell. Interestingly, in electrical engineering, logarithmic and exponential 
amplifiers are paired to compress and then reexpand signals. Following a pulse of MYC-driven global activation, the panoply of induced TFs and chromatin regulators sustain expression as MYC levels decline. Unremittingly high-level MYC expression is principally a pathologic feature of neoplasia and other proliferative diseases.

Sustained high-level MYC expression in tumors may directly and indirectly overdrive cellular networks past their redlines. In this situation, selection for compensatory changes, for example, antiapoptic/prosurvival pathway activation, would be expected to countermand the scaled-up output of apoptic pathways. In addition, although at physiological levels MYC is found at promoters far more than at enhancers (Lin et al. 2012; Nie et al. 2012), at pathological levels, MYC invades enhancers, likely increasing their activity. Besides directly increasing transcriptional output at promoters, relentless MYC action may support epigenetic changes at promoters and enhancers that help to lock cancer cells into their pathologic state via interactions with chromatin-modifying and re-modeling complexes.

The global action of MYC would seem to belie its specific affinity for E-boxes and the consistent enrichment of E-boxes in genomewide in vivo DNA-binding studies. Reconciliation of this E-box paradox has several components. First, the statistical methods used to cull candidate cognate elements from genomewide studies are very powerful. The sheer magnitude of the data and the abundance of binding sites detected, with or without stringent peak-calling thresholds, impart vast statistical significance onto a biochemically modest enrichment. Overall E-box promoters and nonE-box promoters populate the same unimodal distribution, with the former binding $\sim 15 \%$ more MYC on average (Nie et al. 2012). Allowing for degenerate E-boxes, nonspecific binding with DNA and for interactions with the transcription and chromatin machinery, the negative free energy associated with E-box recognition may bias but not license MYC action at its targets. Moreover, the frequency of degenerate E-boxes may be sufficient to assure
MYC recruitment near most transcription start sites.

\section{SETTING MYC LEVELS}

If MYC functions as an exponential amplifier of all expressed genes, achieving proper levels of MYC is crucial for the execution, control, and homeostasis of physiological processes (Levens 2010; Liu and Levens 2006; Wierstra and Alves 2008). Inappropriate levels or fluxes in MYClevels may trigger network instability as all downstream direct and indirect targets seek new set points or become dynamically unstable owing to feed-forward and feedback circuitry. Because MYC influences the abundance of all active genes, it would be expected to change the levels of all of its own regulators. In the case of positive feedback, this would create a potentially lethal situation. To avoid this, MYC is regulated at every possible level of macromolecular synthesis and degradation, from chromatin modification and remodeling through transcription to RNA and protein degradation. As the regulation of MYC protein stability is dealt with elsewhere in this collection (Farrell and Sears 2013), the regulation of MYC transcription will be considered here starting with the definition of the MYC locus. The $6-\mathrm{kb}$ coding region situated on the long arm of chromosome 8 resides in a gene desert $50 \mathrm{~kb}$ upstream of PVT1 and more than a megabase downstream from the nearest upstream gene (Pomerantz et al. 2009; Tuupanen et al. 2009; Sotelo et al. 2010; Kovalchuk et al. 2012; Sur et al. 2012). MYC mRNA is transcribed principally from the weak $\mathrm{P} 1$ promoter and the stronger $\mathrm{P} 2$ promoter $150 \mathrm{bp}$ further downstream; additional minor transcription start sites include P0, 600 bp upstream of $\mathrm{P} 2$ and $\mathrm{P} 3$ in exon 1 as well as others. P1 and P2 reside in constitutive nucleosome-depleted nuclease-hypersensitive sites, as does the CT element (also called nuclease hypersensitive element 1, NHE1) at a sequence with the capacity to assume multiple complex conformations (Liu and Levens 2006). Other hypersensitive sites are associated with induced expression. MYC was among the first genes shown to harbor a transcriptionally engaged RNA polymerase paused 
D. Levens

35-50 nucleotides downstream from the start site, awaiting release into the elongation mode (Spencer and Groudine 1990). Once thought to be rare, it has become apparent that such promoter-paused polymerases may well be the predominant mode of regulation at most active genes (Seila et al. 2009; Levine 2011; Adelman and Lis 2012; Luo et al. 2012). Initial studies of the MYC promoter sought to identify the TFs and their binding sites within the vicinity of the transcription start site (TSS) that were necessary or sufficient to confer proper regulation on a reporter (proper meaning the reporter mimics the expression profile of the endogenous gene). However, such efforts proved to be largely unilluminating. Although the minimal promoter itself could suffer a staggering degree of damage by mutagenesis (including to the TATA-box and INR) while still supporting transcription (Krumm et al. 1995), no regulatory domain could be defined that delimited the sequences required for proper regulation.

\section{THE MYC LOCUS: FINITE BUT UNBOUNDED?}

The current regulatory topography of the MYC locus can be charted by superimposing the in vivo/in situ studies of the native $c$-myc gene using methods such as nuclease hypersensitivity assays, genome-wide chromatin immunoprecipitation (ChIP), chromatin conformation capture (3C), and in vivo footprinting. When combined with in vitro binding and transcription assays using manipulated or reconstructed/ reconstituted regulatory elements and factors, and with reporter assays (transient and stable transfections) and transgenic animal studies, the complexity of MYC regulation is manifest. All of these approaches typically seek to expose the workings of individual pathways among the myriad that converge on the MYC promoter. Most signaling systems impact $c$-myc expression, usually by directly modifying the abundance or activity of one or a few among the host of transcription and chromatin regulatory proteins and complexes that bind to the MYC gene (Fig. 2). Indeed, because MYC is an amplifier, any TF targeting MYC magnifies its own effectiveness, as has been recently shown for the androgen receptor (Ni et al. 2013). Among the regulatory processes influencing $c-m y c$ transcription are hormones, metabolites, intracellular second messengers, kinase cascades, and protein acetylation. A full catalog of the binding sites of all factors and their elements for MYC is beyond the scope of this article, but the reader is referred to reviews by Liu and Levens (2006), Wierstra and Alves (2008), and Levens (2010). Although binding sites for many factors have been mapped, it has been extremely difficult to establish unequivocally the role and significance of many elements. Two issues particularly bedevil these studies. First, many of the factors and pathways impart their influence on $c-m y c$ through multiple compensatory, if not fully redundant, elements distributed across long distances. Most TFs interact with the $c-m y c$ chromosomal domain at multiple sites. For example, TCF was first reported to bind $\sim 1.3 \mathrm{~kb}$ upstream of the TSS (He et al. 1998); subsequently, TSS sites were reported extending from $30 \mathrm{~kb} 3^{\prime}$ to $400 \mathrm{~kb} \mathrm{5}$ of the TSS (Yochum et al. 2008, 2010; Pomerantz et al. 2009; Tuupanen et al. 2009; Sotelo et al. 2010). The best characterized of these is a TCF7L2-binding site that is $400 \mathrm{~kb}$ upstream with a consensus sequence that includes a single-nucleotide polymorphism (SNP) associated with better TCF binding and with colon cancer. This region loops to the promoter, as detected by $3 \mathrm{C}$. The second issue involves the difficulty of assessing the physiological or pathologic function of an element. Deletion of the homologous TCFL2-binding region in mice only slightly lowers MYC mRNA levels in the colon while rendering these animals dramatically refractory to anaphase-promoting complex (APC)-mediated polyposis, consistent with the notion that small increments in MYC expression are associated with dramatic pathologic consequences (Sur et al. 2012). The marginal effect on expression observed experimentally after deletion of this site would have been discounted, lacking the cancer-associated SNP. Whether this SNP is associated with a larger magnitude effect in a subset of colonic cells or the subtle differences in MYC levels observed are sufficient to account for the associated tumori- 


\section{A}
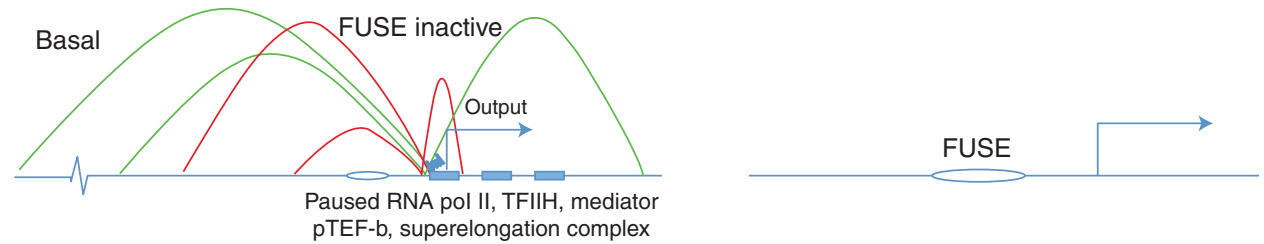

B
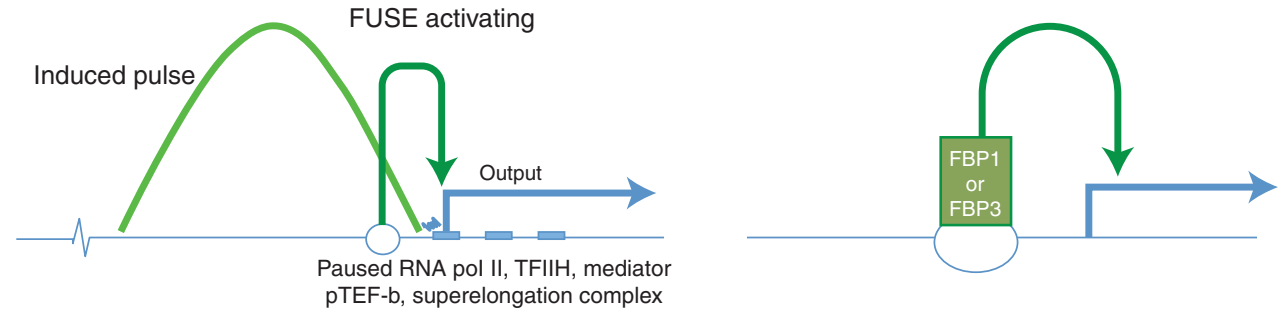

C
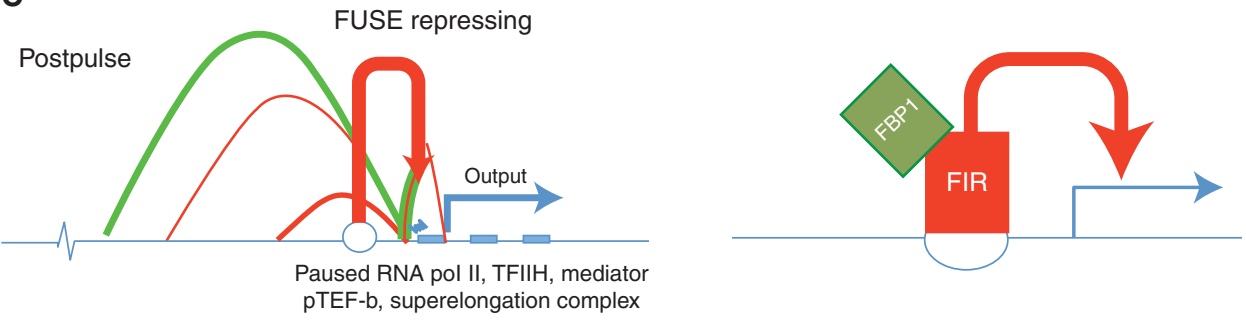

Figure 2. Integrating input at the $c-m y c$ promoter. $(A$, left $) M Y C$ sits in a gene desert removed from other genes and their activating or repressing signals. The $M Y C$ region acts as an antenna picking up positive (green lines) and negative (red lines) inputs that flicker on and off. $(A$, right) The intensity of basal transcriptional output (thin blue line) is too low to generate sufficient mechanical torque to twist open the far upstream element (FUSE). ( $B$, right) When a single signal (thick green line) or the sum of multiple signals (not shown) surmount the torque threshold required to melt FUSE, the FUSE-binding protein (FBP) system kicks in (dark green). ( $B$, left) After melting, FBP or FBP3 (dark green) are recruited to further intensify transcriptional output (thick blue arrow). ( $C$, left ) With more intense MYC expression, some MYC targets, including FBP interacting repressor (FIR) (thick red line), directly or indirectly feed back (red lines) to decrease MYC promoter output and restore basal level transcription (thin blue line). ( $C$, right) FIR is recruited to active FUSE by binding with FBP and single-stranded DNA. With declining transcription, FBP is ejected, but FIR lingers at FUSE to enforce low-level expression (thick red line).

genesis remains unknown. The boundaries of the $c$-myc locus have not been enumerated. The numbers and activities of other remote elements remain to be determined.

\section{FUNNY DNA AS A SENSOR OF c-myc ACTIVITY}

The existence and significance of alternative DNA structures for eukaryotic gene regulation have been largely underexplored and perhaps underappreciated (Sinden 1994). Typically, computational algorithms validated by biophysical studies in vitro, often using single- or double-stranded oligonucleotides, have been used to infer the potential for alternative DNA structures in vivo. However, these algorithms and indeed, the in vitro studies, have only infrequently been validated by in vivo data limiting their general impact. In vivo variables unaccounted for in computations or biophysical studies have the potential to degrade the accu- 


\section{Levens}

racy of the calculations. Foremost among these variables is the status of chromatin-the position and density of nucleosomes-as well as consideration of the binding of other TFs and chromatin regulatory complexes and of the magnitude and dynamics of mechanical forces applied to the genome by genetic processes. The MYC gene has served as a proving ground for strategies and methods to explore the existence and importance of DNA conformational dynamics in health and disease (Brooks and Hurley 2009).

Several regions of $M Y C$ have been predicted and shown to adopt non-B DNA conformations in vitro. Included among these alternatives to the conventional right-handed Watson-Crick B-DNA double helix are melted bubbles (single-stranded loops), Z-DNA (a left-handed double helix with a 12 bp repeat), and quadruplex G4 DNA comprised of intrastrand stacks (usually at least three) of planar rings, each composed of four non-Watson-Crick hydrogenbonded guanosines centered on a potassium ion. The backbone stems of the $\mathrm{G}$ runs can be oriented parallel or antiparallel to each other and are connected by variable length loops, making G4 DNA highly polymorphous. Other non-B structures include triplex H-DNA and extruded cruciform (hairpins).

All alternative DNA structures must compete for existence against the thermodynamic stability of conventional B DNA. In general, formation of non-B structures must be accompanied by the expenditure of energy via helix-destabilizing enzymes such as helicases, translocases, or DNA and RNA polymerases. In transcription, replication, recombination, and repair, the double helix is actively screwed through the active sites of the engaged enzymes. In situations in which the counter-rotation of the DNA relative to the enzyme is hindered, stress accumulates in the DNA backbone just as in a spring twisted from its relaxed state (Liu and Wang 1987; Kouzine and Levens 2007). The energy of negative supercoiling within the DNA backbone facilitates strand separation and structural reorganization. Supercoiling can be stored stably within a fixed topological domain, or it can be dynamically cranked through an open domain by ongoing genetic processes, most commonly transcription (Kouzine et al. 2004, 2008, 2013; Kouzine and Levens 2007). Topoisomerases I and II that break one or both strands of the double helix allow rotation of the helix around the nick or passage of one double-helical segment through another, respectively, removing or limiting accumulated topological stress. Because of the coupling between dynamic supercoiling and DNA structure, alternative conformations have the potential to serve as real-time mechanosensors that report the intensity of gene activity. For non-B DNA to serve as a mechanosensor, some effector molecules must, in turn, respond to the mechanosensor, and feedback regulate the source(s) of the dynamic supercoiling. The $c-m y c$ gene presents several candidate mechanosensing cis elements and their associated trans factors.

The CT element/nuclease hypersensitive element 1 (NHE1) is a structurally complicated GC-rich region 250 bp upstream of $c$-myc promoter P2. The CT element is constitutively nucleosome depleted (Hay et al. 1987; Liu and Levens 2006; Brooks and Hurley 2009; Levens 2010). The CT element has been reported to bind a variety of TFs such as Spl in vitro and has been proposed to form protein-stabilized single-stranded loops, slipped mismatches of its five-repeated segments, G4 and triplex structures. Although in vivo footprinting indicates some sort of conformational disturbance at this element, the patterns are not especially diagnostic of a particular structure (Michelotti et al. 1996). Most likely, the footprints represent the superimposition of several distinct structural populations. Particular states may be enforced preferentially according to the particular transcriptional dynamics and the array of available TFs in a given cell. In B DNA, the CT element presents an array of five Sp1-like binding sites that will dock with $\mathrm{Sp} 1$ or other zinc-finger proteins. As a melted loop, the purine-rich strand of the CTelement binds with CNBP (also called ZNF9), a single-stranded DNA (ssDNA)- or RNA-binding protein, whereas the pyrimidine-rich strand binds hnRNP K. When folded into quadruplex, the G-rich strand can bind nucleolin (Gonzalez et al. 2009). The transcrip- 
tional effector function of nucleolin, if any, has not been elucidated. The complex interplay and competition between alternative DNA conformations and their interactions with CT-binding factors invites the consideration of many hypothetical regulatory modalities that remain largely unproven.

The far upstream element (FUSE) is an ATrich sequence that in the context of the MYC promoter is highly responsive to the unwinding negative supercoiling forces generated by the transcription of downstream sequences. After melting, FUSE binds members of a family of sequence-specific single-strand-binding TFs (the FUSE-binding proteins, FUBPs) as well as the ssDNA-specific FBP interacting repressor (FIR). Both FBP and FIR act through TFIIH downstream from preinitiation complex assembly to control the advancement of the transcription machinery to the point of pause release (Liu et al. 2001, 2006). Once the transcription elicited by any signal surmounts the threshold that melts FUSE, FBP (or FBP3) is recruited to hasten progression to and through pausing. Higher levels of transcription lead to further melting of FUSE, resulting in recruitment of FIR, which in turn drives MYC back to basal levels of expression (Fig. 2).

Proteins that bind non-B DNA often do so through protein motifs associated with RNA binding, such as KH domains and RNA recognition motifs (RRMs) (Braddock et al. 2002; Benjamin et al. 2008; Crichlow et al. 2008; Valverde et al. 2008; Mitchell et al. 2010). In the absence of definitive enumeration of their in vivo targets or of quantitative thermodynamic and kinetic data discriminating their RNA- versus DNA-binding characteristics, it would perhaps be prudent to designate these molecules as single-stranded nucleic-acid-binding proteins (or even simply nucleic-acid-binding proteins). Whereas duplex DNA-binding proteins are presumed to conduct the bona fide decoding of regulatory information from the genome, DNA binding by RNA-binding proteins (or other unconventional DNA-binding proteins) is often presumed to be a nonspecific manifestation of RNA binding; however, imagining the transition states between the primordial RNA and
DNA worlds invites speculation that the first regulatory DNA-binding proteins most likely looked like RNA-binding proteins, whereas the evolution of proteins truly specific for double-stranded DNA likely represents a later evolutionary elaboration. Logically, when the first DNAs were templated by RNA, only RNAbinding proteins were available. Subsequently most of the regulatory duties of these early proteins were ceded to canonical B-DNA-binding TFs, although any functions demanding ssDNAs or secondary structures derived from unpaired strands, might be expected to conserve RNA-binding-like protein partners. The intrinsic rigidity of $\mathrm{B}$ DNA to bending (persistence length $\sim 50 \mathrm{~nm}, 150 \mathrm{bp}$ ) and especially to twisting (torsional persistence length $\sim 70 \mathrm{~nm}$, $210 \mathrm{bp}$ ) would seem to impose a formidable energetic hurdle opposing the assembly of enhanceosomes and the various loops that have been proposed to juxtapose DNA elements across various scales (Lavelle 2009; Levens and Benham 2011). The strategic placement of sequences adopting non-B conformations, with correspondingly different elastic modulii would seem to be a useful tool coupling cis-element function with DNA structure such as at FUSE.

Chromatin must necessarily constrain the role of non-B DNA conformations in the regulation of gene expression. The X-ray crystallographic structures of nucleosomes show complementarity between histone octamer cores and B DNA (Luger et al. 1997; Davey et al. 2002). Alternative DNA structures must be accommodated either by an ill-fitting bulge lifted above the nucleosomal surface or by complete exclusion from nucleosomes, unless the histones undergo a structural transition friendly to other DNA conformations (Mulholland et al. 2012). The CT element is constitutively nucleosomefree, perhaps reflecting an inability to incorporate G4 DNA onto a nucleosome (Michelotti et al. 1996). In contrast, FUSE is nucleosome occluded in $c$-myc-off cells but nucleosomefree in MYC cells (Michelotti et al. 1996). Mobilization of the occluding nucleosome must precede the torsional stress that induces melting of FUSE. Application of torque to a nucleo- 


\section{Levens}

some-embracing FUSE may be accommodated by en bloc nucleosome rotation or shifts in the trajectory of linker regions that protect FUSE from helix-destabilizing stress. The signal and mechanisms mediating mobilization of the FUSE-occluding nucleosome are unknown, although some evidence implicates Swi/Snf chromatin remodeling with the activity of the $M Y C$ promoter (Nagl et al. 2007). There is also some evidence that Z-DNA may occur in the MYC gene, but the regulatory consequences and in vivo significance of $Z$ formation at any of its three proposed sites remain to be explored (Wolfl et al. 1995, 1997).

The overview that emerges indicates that the MYC promoter is a remarkably robust system comprised of various parallel signaling modules distributed across a vast chromosomal expanse, seemingly isolated from interfering elements from other genes. These modules operate individually and independently when driven by single signals in experimental systems. The ensemble operation of the system in the face of a complex set of physiological and/or pathologic and environmental stimuli remains largely unexplored. The degree to which signals synergize and antagonize and the rules of integration at the MYC promoter remain unknown. The existence of FUSE, CT, and Z-DNA elements suggests that the promoter output is coupled not only with chromatin conformation and dynamics, but also with fundamental changes in DNA secondary structure. In the same way that transcription pausing at the MYC promoter presaged almost global regulation by pause release, MYC may also herald the dynamic coupling between DNA conformation and topology with gene activity on a genome-wide scale. Such mechanisms may prove especially important at genes where fast and coarse fluctuations are likely to prove especially deleterious, such as some oncogenes and tumor suppressors.

\section{ACKNOWLEDGMENTS}

I thank all of my colleagues and the participants at the Banbury meeting on MYC in the fall of 2011 for insights, criticism, and stimulating discussions.

\section{REFERENCES}

*Reference is also in this collection.

Abrams HD, Rohrschneider LR, Eisenman RN. 1982. Nuclear location of the putative transforming protein of avian myelocytomatosis virus. Cell 29: 427-439.

Adelman K, Lis JT. 2012. Promoter-proximal pausing of RNA polymerase II: Emerging roles in metazoans. Nat Rev Genet 13: 720-731.

Agrawal P, Yu K, Salomon AR, Sedivy JM. 2010. Proteomic profiling of Myc-associated proteins. Cell Cycle 9: 49084921.

Alitalo K, Schwab M, Lin CC, Varmus HE, Bishop JM. 1983. Homogeneously staining chromosomal regions contain amplified copies of an abundantly expressed cellular oncogene (c-myc) in malignant neuroendocrine cells from a human colon carcinoma. Proc Natl Acad Sci 80: 17071711.

Alon U. 2007. An introduction to systems biology: Design principles of biological circuits. Chapman \& Hall/CRC, Boca Raton, FL.

Amati B, Frank SR, Donjerkovic D, Taubert S. 2001. Function of the c-Myc oncoprotein in chromatin remodeling and transcription. Biochim Biophys Acta 1471: M135M145.

Benjamin LR, Chung HJ, Sanford S, Kouzine F, Liu J, Levens D. 2008. Hierarchical mechanisms build the DNA-binding specificity of FUSE binding protein. Proc Natl Acad Sci 105: 18296-18301.

Blackwood EM, Eisenman RN. 1991. Max: A helix-loophelix zipper protein that forms a sequence-specific DNA-binding complex with Myc. Science 251: 12111217.

Boxer LM, Dang CV. 2001. Translocations involving $c-m y c$ and $c$-myc function. Oncogene 20: 5595-5610.

Braddock DT, Baber JL, Levens D, Clore GM. 2002. Molecular basis of sequence-specific single-stranded DNA recognition by KH domains: Solution structure of a complex between hnRNP K KH3 and single-stranded DNA. EMBO J 21: 3476-3485.

Brooks TA, Hurley LH. 2009. The role of supercoiling in transcriptional control of MYC and its importance in molecular therapeutics. Nat Rev Cancer 9: 849-861.

Cowling VH, Cole MD. 2006. Mechanism of transcriptional activation by the Myc oncoproteins. Semin Cancer Biol 16: $242-252$.

Cowling VH, Cole MD. 2007. The Myc transactivation domain promotes global phosphorylation of the RNA polymerase II carboxy-terminal domain independently of direct DNA binding. Mol Cell Biol 27: 2059-2073.

Crichlow GV, Zhou H, Hsiao HH, Frederick KB, Debrosse M, Yang Y, Folta-Stogniew EJ, Chung HJ, Fan C, De la Cruz EM, et al. 2008. Dimerization of FIR upon FUSE DNA binding suggests a mechanism of $c-m y c$ inhibition. EMBO J 27: 277-289.

Dang CV. 2010. Enigmatic MYC conducts an unfolding systems biology symphony. Genes Cancer 1: 526-531.

Dang CV. 2012. MYC on the path to cancer. Cell 149: 22-35.

Davey CA, Sargent DF, Luger K, Maeder AW, Richmond TJ. 2002. Solvent mediated interactions in the structure of 
the nucleosome core particle at 1.9 a resolution. $J$ Mol Biol 319: 1097-1113.

de la Cova C, Abril M, Bellosta P, Gallant P, Johnston LA. 2004. Drosophila Myc regulates organ size by inducing cell competition. Cell 117: 107-116.

Eilers M, Eisenman RN. 2008. Myc's broad reach. Genes Dev 22: 2755-2766.

Eilers M, Picard D, Yamamoto KR, Bishop JM. 1989. Chimaeras of myc oncoprotein and steroid receptors cause hormone-dependent transformation of cells. Nature 340: $66-68$.

* Eisenman R. 2013. An overview of MYC and its interactome. Cold Spring Harb Perspect Med doi: 10.1101/cshperspect.a014357.

* Farrell AS, Sears RC. 2013. MYC degradation. Cold Spring Harb Perspect Med doi: 10.1101/cshperspect.a014365.

Gonzalez V, Guo K, Hurley L, Sun D. 2009. Identification and characterization of nucleolin as a c-myc G-quadruplex-binding protein. J Biol Chem 284: 23622-23635.

Guney I, Wu S, Sedivy JM. 2006. Reduced c-Myc signaling triggers telomere-independent senescence by regulating Bmi-1 and p16 ${ }^{\mathrm{INK} 4 \mathrm{a}}$. Proc Natl Acad Sci 103: 3645-3650.

Hay N, Bishop JM, Levens D. 1987. Regulatory elements that modulate expression of human C-Myc. Genes Dev 1: 659-671.

He TC, Sparks AB, Rago C, Hermeking H, Zawel L, da Costa LT, Morin PJ, Vogelstein B, Kinzler KW. 1998. Identification of c-MYC as a target of the APC pathway. Science 281: $1509-1512$.

Iritani BM, Eisenman RN. 1999. c-Myc enhances protein synthesis and cell size during B lymphocyte development. Proc Natl Acad Sci 96: 13180-13185.

Janz S. 2006. Myc translocations in B cell and plasma cell neoplasms. DNA Repair (Amst) 5: 1213-1224.

Ji H, Wu G, Zhan X, Nolan A, Koh C, De Marzo A, Doan HM, Fan J, Cheadle C, Fallahi M, et al. 2011. Cell-type independent MYC target genes reveal a primordial signature involved in biomass accumulation. PLOS ONE 6: e26057.

* Johnston LA. 2013. Socializing with Myc: Cell competition in develoment and as a model for premalignant cancer. Cold Spring Harb Perspect Med doi: 10.1101/cshperspect.a014274.

Johnston LA, Prober DA, Edgar BA, Eisenman RN, Gallant P. 1999. Drosophila myc regulates cellular growth during development. Cell 98: 779-790.

Kaur M, Cole MD. 2013. MYC acts via the PTEN tumor suppressor to elicit autoregulation and genome-wide gene repression by activation of the Ezh2 methyltransferase. Cancer Res 73: 695-705.

Kim J, Woo AJ, Chu J, Snow JW, Fujiwara Y, Kim CG, Cantor AB, Orkin SH. 2010. A Myc network accounts for similarities between embryonic stem and cancer cell transcription programs. Cell 143: 313-324.

Kouzine F, Levens D. 2007. Supercoil-driven DNA structures regulate genetic transactions. Front Biosci 12: 4409-4423.

Kouzine F, Liu JH, Sanford S, Chung HJ, Levens D. 2004 The dynamic response of upstream DNA to transcription-generated torsional stress. Nat Struct Mol Biol 11: $1092-1100$.
Kouzine F, Sanford S, Elisha-Feil Z, Levens D. 2008. The functional response of upstream DNA to dynamic supercoiling in vivo. Nat Struct Mol Biol 15: 146-154.

Kouzine F, Wojtowicz D, Yamane A, Resch W, Kieffer-Kwon K-R, Bandle R, Nelson S, Nakahashi H, Awasthi P, Feigenbaum L, et al. 2013. Global regulation of promoter melting in naive lymphocytes. Cell 153: 988-999.

Kovalchuk AL, Ansarah-Sobrinho C, Hakim O, Resch W, Tolarova H, Dubois W, Yamane A, Takizawa M, Klein I, Hager GL, et al. 2012. Mouse model of endemic Burkitt translocations reveals the long-range boundaries of Igmediated oncogene deregulation. Proc Natl Acad Sci 109: 10972-10977.

Krumm A, Hickey LB, Groudine M. 1995. Promoterproximal pausing of RNA polymerase II defines a general rate-limiting step after transcription initiation. Genes Dev 9: 559-572.

Lavelle C. 2009. Forces and torques in the nucleus: Chromatin under mechanical constraints. Biochem Cell Biol 87: 307-322.

Lebel R, McDuff FO, Lavigne P, Grandbois M. 2007. Direct visualization of the binding of $\mathrm{c}-\mathrm{Myc} / \mathrm{Max}$ heterodimeric b-HLH-LZ to E-box sequences on the hTERT promoter. Biochemistry 46: 10279-10286.

Levens D. 2010. You don't muck with Myc. Genes Cancer 1: 547-554.

Levens D, Benham CJ. 2011. DNA stress and strain, in silico, in vitro and in vivo. Phys Biol 8: 035011

Levine M. 2011. Paused RNA polymerase II as a developmental checkpoint. Cell 145: 502-511.

Lin CY, Loven J, Rahl PB, Paranal RM, Burge CB, Bradner JE, Lee TI, Young RA. 2012. Transcriptional amplification in tumor cells with elevated c-Myc. Cell 151: 56-67.

Littlewood TD, Hancock DC, Danielian PS, Parker MG, Evan GI. 1995. A modified oestrogen receptor ligandbinding domain as an improved switch for the regulation of heterologous proteins. Nucleic Acids Res 23: 16861690.

Liu J, Levens D. 2006. Making myc. Curr Top Microbiol Immunol 302: 1-32.

Liu LF, Wang JC. 1987. Supercoiling of the DNA-template during transcription. Proc Natl Acad Sci 84: 7024-7027.

Liu JH, Akoulitchev S, Weber A, Ge H, Chuikov S, Libutti D, Wang XW, Conaway JW, Harris CC, Conaway RC, et al. 2001. Defective interplay of activators and repressors with TFIIH in xeroderma pigmentosum. Cell 104: 353-363.

Liu J, Kouzine F, Nie Z, Chung HJ, Elisha-Feil Z, Weber A, Zhao K, Levens D. 2006. The FUSE/FBP/FIR/TFIIH system is a molecular machine programming a pulse of c-myc expression. EMBO J 25: 2119-2130.

Love C, Sun Z, Jima D, Li G, Zhang J, Miles R, Richards KL, Dunphy CH, Choi WW, Srivastava G, et al. 2012. The genetic landscape of mutations in Burkitt lymphoma. Nat Genet 44: 1321-1325.

Loven J, Orlando DA, Sigova AA, Lin CY, Rahl PB, Burge CB, Levens DL, Lee TI, Young RA. 2012. Revisiting global gene expression analysis. Cell 151: 476-482.

Luger K, Mader AW, Richmond RK, Sargent DF, Richmond TJ. 1997. Crystal structure of the nucleosome core particle at 2.8 A resolution. Nature 389: 251-260. 
D. Levens

Luo Z, Lin C, Shilatifard A. 2012. The super elongation complex (SEC) family in transcriptional control. Nat Rev Mol Cell Biol 13: 543-547.

Margolin AA, Palomero T, Sumazin P, Califano A, Ferrando AA, Stolovitzky G. 2009. ChIP-on-chip significance analysis reveals large-scale binding and regulation by human transcription factor oncogenes. Proc Natl Acad Sci 106: 244-249.

Michelotti GA, Michelotti EF, Pullner A, Duncan RC, Eick D, Levens D. 1996. Multiple single-stranded cis elements are associated with activated chromatin of the human c-myc gene in vivo. Mol Cell Biol 16: 2656-2669.

Mitchell NC, Johanson TM, Cranna NJ, Er AL, Richardson HE, Hannan RD, Quinn LM. 2010. Hfp inhibits Drosophila Myc transcription and cell growth in a TFIIH/Haydependent manner. Development 137: 2875-2884.

Moelling K, Owada MK, Greiser-Wilke I, Bunte T, Donner P. 1982. Biochemical characterization of transformationspecific proteins of acute avian leukemia and sarcoma viruses. J Cell Biochem 20: 63-69.

Moreno E, Basler K. 2004. dMyc transforms cells into supercompetitors. Cell 117: 117-129.

Mulholland N, Xu Y, Sugiyama H, Zhao K. 2012. SWI/SNFmediated chromatin remodeling induces Z-DNA formation on a nucleosome. Cell Biosci 2: 3.

Murphy DJ, Junttila MR, Pouyet L, Karnezis A, Shchors K, Bui DA, Brown-Swigart L, Johnson L, Evan GI. 2008. Distinct thresholds govern Myc's biological output in vivo. Cancer Cell 14: 447-457.

Nagl NG Jr, Wang X, Patsialou A, Van Scoy M, Moran E. 2007. Distinct mammalian SWI/SNF chromatin remodeling complexes with opposing roles in cell-cycle control. EMBO J 26: 752-763.

Nair SK, Burley SK. 2003. X-ray structures of Myc-Max and Mad-Max recognizing DNA. Molecular bases of regulation by proto-oncogenic transcription factors. Cell 112: 193-205.

Ni M, Chen Y, Fei T, Li D, Lim E, Liu XS, Brown M. 2013. Amplitude modulation of androgen signaling by c-MYC. Genes Dev 27: 734-748.

Nie Z, Hu G, Wei G, Cui K, Yamane A, Resch W, Wang R, Green DR, Tessarollo L, Casellas R, et al. 2012. c-Myc is a universal amplifier of expressed genes in lymphocytes and embryonic stem cells. Cell 151: 68-79.

O'Connell BC, Cheung AF, Simkevich CP, Tam W, Ren X, Mateyak MK, Sedivy JM. 2003. A large scale genetic analysis of c-Myc-regulated gene expression patterns. J Biol Chem 278: 12563-12573.

Park S, Chung S, Kim KM, Jung KC, Park C, Hahm ER, Yang CH. 2004. Determination of binding constant of transcription factor myc-max/max-max and E-box DNA: The effect of inhibitors on the binding. Biochim Biophys Acta 1670: 217-228.

Peter M, Rosty C, Couturier J, Radvanyi F, Teshima H, Sastre-Garau X. 2006. MYC activation associated with the integration of HPV DNA at the MYC locus in genital tumors. Oncogene 25: 5985-5993.

Pomerantz MM, Ahmadiyeh N, Jia L, Herman P, Verzi MP, Doddapaneni H, Beckwith CA, Chan JA, Hills A, Davis M, et al. 2009. The 8q24 cancer risk variant rs6983267 shows long-range interaction with MYC in colorectal cancer. Nat Genet 41: 882-884.

* Rahl PB, Young RA. 2013. Myc and transcription elongation. Cold Spring Harb Perspect Med doi: 10.1101/cshperspect.a014340.

Rahl PB, Lin CY, Seila AC, Flynn RA, McCuine S, Burge CB, Sharp PA, Young RA. 2010. c-Myc regulates transcriptional pause release. Cell 141: 432-445.

Schuhmacher M, Staege MS, Pajic A, Polack A, Weidle UH Bornkamm GW, Eick D, Kohlhuber F. 1999. Control of cell growth by c-Myc in the absence of cell division. Curr Biol 9: 1255-1258.

Seila AC, Core LJ, Lis JT, Sharp PA. 2009. Divergent transcription: A new feature of active promoters. Cell Cycle 8: $2557-2564$.

Shachaf CM, Gentles AJ, Elchuri S, Sahoo D, Soen Y, Sharpe O, Perez OD, Chang M, Mitchel D, Robinson WH, et al. 2008. Genomic and proteomic analysis reveals a threshold level of MYC required for tumor maintenance. Cancer Res 68: 5132-5142.

Shaffer AL, Wright G, Yang L, Powell J, Ngo V, Lamy L, Lam LT, Davis RE, Staudt LM. 2006. A library of gene expression signatures to illuminate normal and pathological lymphoid biology. Immunol Rev 210: 67-85.

Shrivastava A, Yu J, Artandi S, Calame K. 1996. YY1 and cMyc associate in vivo in a manner that depends on c-Myc levels. Proc Natl Acad Sci 93: 10638-10641.

Sinden RR. 1994. DNA structure and function. Academic Press, San Diego.

Sotelo J, Esposito D, Duhagon MA, Banfield K, Mehalko J, Liao H, Stephens RM, Harris TJ, Munroe DJ, Wu X. 2010. Long-range enhancers on 8q24 regulate c-Myc. Proc Natl Acad Sci 107: 3001-3005.

Spencer CA, Groudine M. 1990. Transcription elongation and eukaryotic gene regulation. Oncogene 5: 777-785.

Sur IK, Hallikas O, Vaharautio A, Yan J, Turunen M, Enge M, Taipale M, Karhu A, Aaltonen LA, Taipale J. 2012. Mice lacking a Myc enhancer that includes human SNP rs6983267 are resistant to intestinal tumors. Science 338: $1360-1363$.

Trumpp A, Refaeli Y, Oskarsson T, Gasser S, Murphy M, Martin GR, Bishop JM. 2001. c-Myc regulates mammalian body size by controlling cell number but not cell size. Nature 414: 768-773.

Tuupanen S, Turunen M, Lehtonen R, Hallikas O, Vanharanta S, Kivioja T, Bjorklund M, Wei G, Yan J, Niittymaki I, et al. 2009. The common colorectal cancer predisposition SNP rs6983267 at chromosome 8q24 confers potential to enhanced Wnt signaling. Nat Genet 41: 885-890.

Valverde R, Edwards L, Regan L. 2008. Structure and function of KH domains. FEBS J 275: 2712-2726.

Vennstrom B, Sheiness D, Zabielski J, Bishop JM. 1982. Isolation and characterization of $c-m y c$, a cellular homo$\log$ of the oncogene $(v-m y c)$ of avian myelocytomatosis virus strain 29. J Virol 42: 773-779.

Walz S, Eilers M. 2013. Unlocking the mysterious mechanisms of Myc. Nat Med 19: 26-27.

Wasylishen AR, Penn LZ. 2010. Myc: The beauty and the beast. Genes Cancer 1: 532-541.

Wierstra I, Alves J. 2008. The $c-m y c$ promoter: Still MysterY and challenge. Adv Cancer Res 99: 113-333. 


\section{Cellular MYCro Economics}

Wolfl S, Wittig B, Rich A. 1995. Identification of transcriptionally induced Z-DNA segments in the human c-myc gene. Biochim Biophys Acta 1264: 294-302.

Wolfl S, Wittig B, Dorbic T, Rich A. 1997. Identification of processes that influence negative supercoiling in the human c-myc gene. Biochim Biophys Acta 1352: 213221.

Yap CS, Peterson AL, Castellani G, Sedivy JM, Neretti N 2011. Kinetic profiling of the c-Myc transcriptome and bioinformatic analysis of repressed gene promoters. Cell Cycle 10: 2184-2196.

Yochum GS, Cleland R, Goodman RH. 2008. A genomewide screen for $\beta$-catenin binding sites identifies a downstream enhancer element that controls c-Myc gene expression. Mol Cell Biol 28: 7368-7379.

Yochum GS, Sherrick CM, Macpartlin M, Goodman RH. 2010. A $\beta$-catenin/TCF-coordinated chromatin loop at MYC integrates $5^{\prime}$ and $3^{\prime}$ Wnt responsive enhancers. Proc Natl Acad Sci 107: 145-150. 


\section{$\&_{\mathrm{CSH}}^{\infty} \&$ Cold Spring Harbor

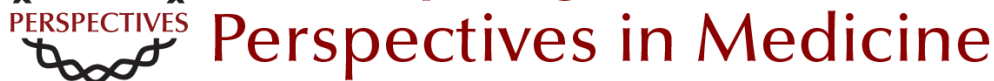

\section{Cellular MYCro Economics: Balancing MYC Function with MYC Expression}

David Levens

Cold Spring Harb Perspect Med 2013; doi: 10.1101/cshperspect.a014233

Subject Collection MYC and the Pathway to Cancer

MYC Cofactors: Molecular Switches Controlling

Diverse Biological Outcomes Stephen R. Hann

MYC Association with Cancer Risk and a New Model of MYC-Mediated Repression Michael D. Cole

MYC and the Art of MicroRNA Maintenance James N. Psathas and Andrei Thomas-Tikhonenko

MYC Activation Is a Hallmark of Cancer Initiation and Maintenance Meital Gabay, Yulin Li and Dean W. Felsher

MYC and Mitochondrial Biogenesis Fionnuala Morrish and David Hockenbery

Synthetic Lethal Screens as a Means to Understand and Treat MYC-Driven Cancers Silvia Cermelli, In Sock Jang, Brady Bernard, et al.

An Overview of MYC and Its Interactome Maralice Conacci-Sorrell, Lisa McFerrin and Robert N. Eisenman

Socializing with MYC: Cell Competition in Development and as a Model for Premalignant Cancer

Laura A. Johnston
MYC and the Control of Apoptosis Steven B. McMahon

Therapeutic Strategies to Inhibit MYC Michael R. McKeown and James E. Bradner

MYC and the Control of DNA Replication David Dominguez-Sola and Jean Gautier

MYC Regulation of Cell Growth through Control of Transcription by RNA Polymerases I and III Kirsteen J. Campbell and Robert J. White

MYC Degradation Amy S. Farrell and Rosalie C. Sears

MYC and Transcription Elongation Peter B. Rahl and Richard A. Young

c-MYC-Induced Genomic Instability Alexandra Kuzyk and Sabine Mai

Oncogenic Mechanisms in Burkitt Lymphoma Roland Schmitz, Michele Ceribelli, Stefania Pittaluga, et al.

For additional articles in this collection, see http://perspectivesinmedicine.cshlp.org/cgi/collection/ 